\title{
Review of MEMS differential scanning calorimetry for biomolecular study
}

(C) The Author(s) 2017. This article is published with open access at link.springer.com and journal.hep.com.cn

\begin{abstract}
Differential scanning calorimetry (DSC) is one of the few techniques that allow direct determination of enthalpy values for binding reactions and conformational transitions in biomolecules. It provides the thermodynamics information of the biomolecules which consists of Gibbs free energy, enthalpy and entropy in a straightforward manner that enables deep understanding of the structure function relationship in biomolecules such as the folding/unfolding of protein and DNA, and ligand bindings. This review provides an up to date overview of the applications of DSC in biomolecular study such as the bovine serum albumin denaturation study, the relationship between the melting point of lysozyme and the scanning rate. We also introduce the recent advances of the development of micro-electro-mechanic-system (MEMS) based DSCs.
\end{abstract}

Keywords differential scanning calorimetry, biomolecule, MEMS, thermodynamic

\section{Introduction}

Differential scanning calorimetry (DSC) as a thermoanalytical technique was developed by Watson and O'Neill in 1966 [1]. Since then, a lot of research has been focused on developing high throughput, high sensitivity DSC and

Received November 6, 2016; accepted March 9, 2017

Shifeng YU, Lei ZUO ( $($ )

Department of Mechanical Engineering, Virginia Tech, Blacksburg, VA 24061, USA

E-mail: leizuo@vt.edu

Shuyu WANG

Department of Mechanical Engineering, Stony Brook University, Stony Brook, NY 11794, USA

Ming LU

Center for Functional Nanomaterials, Brookhaven National Laboratory, Upton, NY 11973, USA applying it into different research areas such as polymer study, biomolecular study, and drug design. A differential scanning calorimeter consists of twin cells (sample and reference) in which temperature sensor and heating module are integrated and operate in differential mode. During a differential temperature scanning process, the difference in temperature (power consumption) between the sample and reference material is measured as a function of linear temperature cycle. The difference directly reveals the heat release or absorption of the sample over temperature which further indicates the heat capacity change. There are mainly two types of DSCs based on the working principle: Power compensated DSC and heat flux DSC. For the power compensated DSC, the temperature of the sample and reference material are always kept the same by varying the heat flow to the sample and reference during the linear temperature scanning process. While in a heat flux DSC, the temperature difference is directly recorded during the same procedure. Together with the thermal resistance, the temperature difference can be converted to the heat flow difference [2]. Recently, another type of DSC was developed which is called temperature modulated differential scanning calorimetry (TMDSC) or alternative current differential scanning calorimetry (AC DSC) [35]. The basic idea of the TMDSC is to add a controlled temperature modulation to the conventional linear heating. The heating process of TMDSC can be divided into two parts. The first part is to heat the sample at a certain temperature scanning rate just like the conventional DSC. In the second part, the heat capacity component of the heat flow is obtained by applying a controlled oscillating temperature modulation with a zero net temperaturechange. In a TMDSC thermal analysis, the average scanning rate, the period of modulation and the temperature amplitude of modulation are three important variables that are tuned to optimize the experiment.

Virtually all biological phenomena depend on molecular interaction. A basic biology problem is to understand the folding and denaturation processes of a protein, i.e., the kinetics and thermodynamics how a protein unfolds and 
folds back into its native state [6]. Both folding/unfolding and denaturation processes are associated with enthalpy changes. The intermolecular interactions such as proteinligand association, protein/DNA interaction [7,8], antigenantibody binding processes are either enthalpically or entropically driven, depending upon the binding modes of the small molecules [9]. The determination of the affinity thermodynamics of binding compounds helps greatly to understand the nature of such molecules [10]. The specificity of binding reactions has fascinated biologists from the very beginning to quantitatively describe the driving forces that govern the formation of biomolecule [11]. This has great significance to the development of vaccines, new drugs and other molecular compounds [12]. There is a rapid growing number of high resolution crystal structures of biomolecules. However, the theoretical concepts which are developed in the tradition of physical-organic chemistry is not enough to understand such driving force of the large complex biomolecular structure straightforwardly. The proteins behave cooperatively and undergo structural rearrangements during the binding reactions. Such binding process has a complicated energy profile involving different energetic expenditures in going from free components to the final complex which can be detected directly by DSC [13].

There are several commercial DSCs for large biomolecular study. One is the MicroCal VP-DSC developed by Malvern. It can directly measure the intramolecular stability of structured macromolecules as well as the intermolecular stability of complexes such as proteins, nucleic acid duplexes and lipid and detergent micellar systems. It consumes $700 \mu \mathrm{L}$ sample for each test and has a high enthalpy resolution as $0.01 \mathrm{~J} /{ }^{\circ} \mathrm{C}$. The sample concentration typically ranges from 0.1 to $2 \mathrm{mg} / \mathrm{mL}$. As a capillary DSC, it is important to clean the reservoir each time after the test and this limits the concentration of the bio-sample since high concentration protein sample would stick on the wall of the capillary after denaturation and it is hard to be washed away. The maximum scanning rate of the MicroCal VP-DSC is only $1.5^{\circ} \mathrm{C} / \mathrm{min}$ which leads to relatively low throughput. Another typical commercial DSC is the TA DSC. As the most cost-effective DSC in industry. It has a different sample preparation strategy compared to the MicroCal VP-DSC. Unlike the VP-DSC which use a syringe to inject the liquid sample to the reservoir through a capillary system, the TA DSC utilizes aluminum pan for the sample preparation and sealing. The sample pan and reference pan are then put on the pedestals in the test chamber for temperature scanning. It can measure protein sample with high concentration as 100 $\mathrm{mg} / \mathrm{mL}$. It can also be used to conduct thermoanalysis of polymers, solid crystals, etc. One limit of the TA DSC is its relatively low temperature accuracy $\left(0.025{ }^{\circ} \mathrm{C}\right.$ for DSC 2500). Figure 1 shows two commercial DSCs [14,15].

Although the commercial DSC can provide high repeatability and reliability for biomolecular study, one major drawback of such macroscale DSC is the large thermal mass of the calorimetric cell itself and its associated hardware. Consequently, the required energy input may be large compared with the energy associated with the thermal transitions to be measured. Hence, they usually require large amount of sample. This can pose problems in cases where only minute sample quantities are available for testing. There has been a strong demand to develop high throughput and high sensitivity calorimeter for rapid detection of biomolecular interaction for decades [16-19]. The microfabrication and nanofabrication technologies allow the miniaturization of the conventional bench-top scale instrument for the building of micro/nano DSC with microfluidic embedded systems, micro temperature sensors and heaters [20]. Such miniaturized DSC has many advantages compare to the current commercial

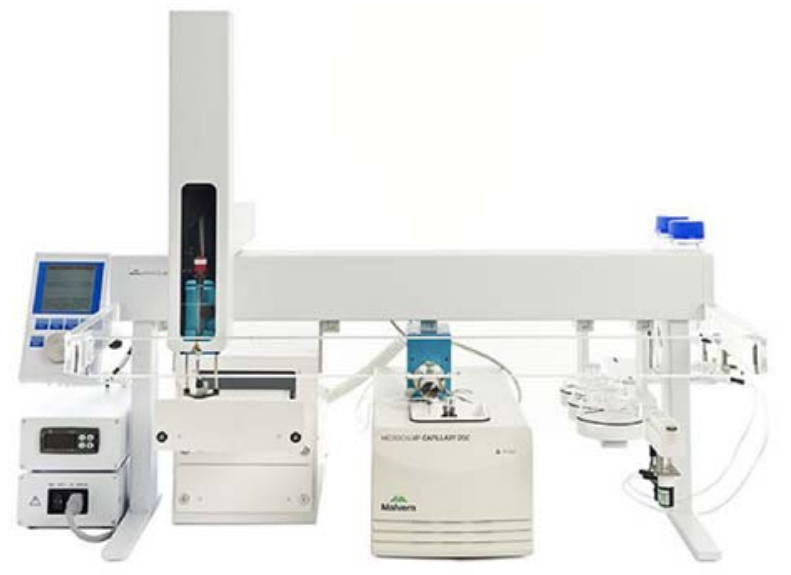

(a)

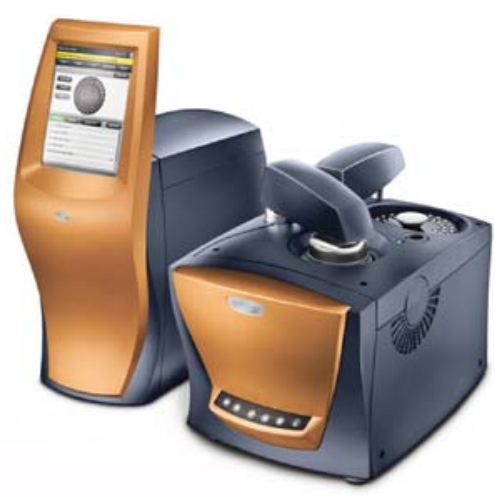

(b)

Fig. 1 Two typical commercial DSCs. (a) The MicroCal VP-DSC, it consists of the sample array, robotic arm for sample handling and the temperature scanning system [14]; (b) the TA DSC, it consists of a sample tray, robotic arm for sample handling and the chamber for thermal analysis [15] 
DSC such as parallel operation, less sample consumption, and higher sensitivity. The current commercial DSC consumes tens to hundreds of minutes to test one sample. It would take months to do the thermal analysis for 1000 compound samples with the current commercial DSC in drug development industry. The micro-electro-mechanicsystem (MEMS) based DSC has the potential to shorten the measurement time from months to days by offering higher scanning rate and parallel test. The MEMS based calorimeter usually consumes micro littler or even nanolitle of sample per test which is more than 10 times less than the commercial DSC [21]. This can help to save the cost for biomolecular sample consumption. Protein samples such as MAN-9 Glycan would cost 43 billion $\mathrm{USD} / \mathrm{mol}$, the miniaturized MEMS DSC would help to save a lot of budget in the study of such expensive compound. On the other hand, since the sample consumption is much less, the total thermal mass of the MEMS DSC is significantly reduced which permits higher scanning rate $[22,23]$. The miniaturized DSC unit will also allow us to integrate multiple DSC on one silicon wafer or polymer substrate. There were already some attempts to develop such calorimeter array [18,24,25].

In this review, we will firstly discuss about the basic thermodynamic relationship in the binding process of biomolecular sample during the temperature scanning test, then typical DSC thermogram will be analyzed. We will review the most up to date progress in the development of MEMS based DSC and its challenges at last.

\section{Thermodynamics in DSC test}

Differential scanning calorimetry can characterize the complete thermodynamic profile for both intermolecular binding and intramolecular folding by measuring the enthalpy $\Delta H$, specific heat $\Delta C_{\mathrm{p}}$, and the melting temperature $T_{\mathrm{m}}$. The thermal model is shown in Fig. 2. In Fig. 2(b), $T_{0}$ is the temperature of the environment. $C_{\mathrm{s}}$ and $C_{\mathrm{r}}$ are the heat capacity of the sample and reference material respectively (in the real test, it will also include part of the heat capacity of the material holder). $P_{\mathrm{s}}$ and $P_{\mathrm{r}}$ are the heat flux to the sample and reference respectively to achieve linear temperature scanning. $G_{\mathrm{s}}$ is the heat conduction between the sample cell and the environment while $G_{\mathrm{r}}$ is the heat conduction between the reference cell and the environment. $G_{0}$ is the heat conduction between the sample cell and reference cell.

Controlled heat flux is added both to the reference and sample material to achieve linear temperature scanning. Part of the heat flux is absorbed by the tested material, the other is lost to the surrounding air by convection, conduction and radiation and most of the heat loss is through heat convection in typical DSC test. The thermal equation for heating single unit is expressed in Eq. (1)

$$
C \frac{\mathrm{d} T}{\mathrm{~d} t}+G\left(T-T_{0}\right)=P,
$$

where $C$ is the heat capacity of the heating sample, $G$ is the thermal conductance, which can be expressed by $h \cdot s$ where $h$ is the coefficient of thermal convection and $s$ is the effective area of thermal convection since convection is the main heat transfer mechanism in a DSC test, $T$ is the realtime temperature of the sample, and $T_{0}$ is the temperature of the environment. Generally, $T_{0}$ can be regarded constant then, the system is first order system. For MEMS DSC, the sample consumption is from nanoliter to microliter which means the heat capacity would be quite small. To achieve high sensitivity, it is essential to increase the thermal insulation of the system which means minimize the thermal conductance $G$. In general, $G_{0}$ is small enough to be neglected then we can have Eq. (2) [26]:

$$
\left(C_{\mathrm{s}}-C_{\mathrm{r}}\right) \frac{\mathrm{d} T}{\mathrm{~d} t}+\left(C_{\mathrm{s}}-C_{\mathrm{r}}\right)\left(T-T_{0}\right)=P_{\text {diff }},
$$

where $P_{\text {diff }}$ is the differential heat flux between the sample and reference material which is recorded directly during the scanning test. In Eq. (2), $\left(C_{\mathrm{s}}-C_{\mathrm{r}}\right)\left(T-T_{0}\right)$ is a linear

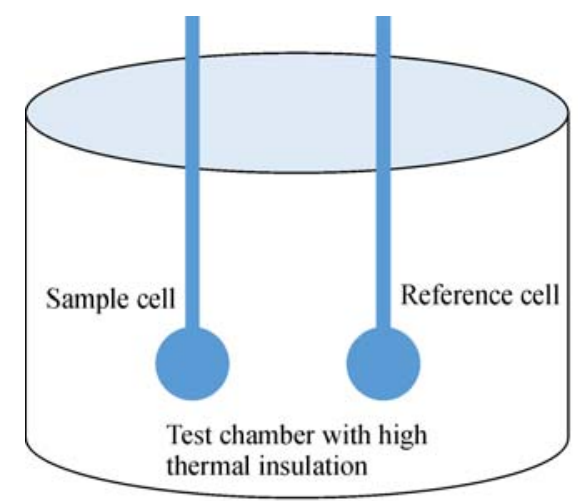

(a)

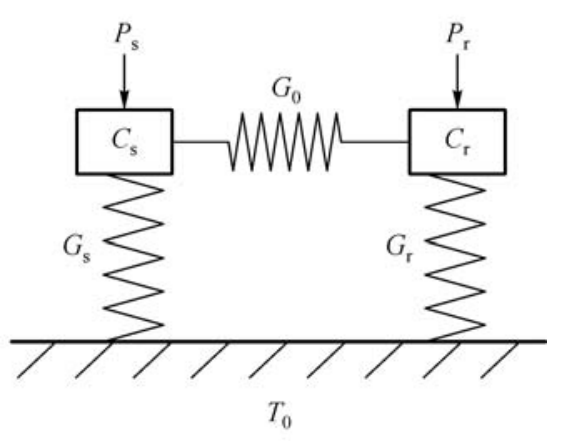

(b)

Fig. 2 The general model for DSC. (a) The schematic diagram for DSC which consists of sample and reference cells (the cells are located in a well-designed test chamber); (b) the thermal model for the DSC 
part and can be removed simply by baseline subtraction, $\mathrm{d} T / \mathrm{d} t$ is the scanning rate which is fixed during the linear temperature scanning process. A temperature domain differential heat capacity between the biomolecular sample and reference can be evaluate by dividing the differential heat flux $P_{\text {diff }}$ by the scanning rate after baseline subtraction. To obtain the heat capacity of the sample, precise knowledge of the reference material (chemical buffer for biomolecular test) is needed for the correction. The differential power $P_{\text {diff }}$ is usually small. In MEMS based DSC, the sample consumption is from nanoliter to microliter, higher concentration is needed to get detectable $P_{\text {diff }}$ compared to the current commercial DSC. More thermodynamic parameter of the biomolecule during the reaction such as denaturation and binding in the scanning process can be derived from the heat capacity change.

Among all the thermodynamic parameters, the Gibbs free energy change $\Delta G$ is the key parameter since its value under particular reactant concentrations dictates the direction of biomolecular equilibria and it is the balance between enthalpy and entropy [27]. It is temperature depended and can be described by Eq. (3).

$$
\Delta G=\Delta H\left(T_{\mathrm{c}}\right)+\int \Delta C \mathrm{~d} T-T \Delta S\left(T_{\mathrm{c}}\right)-T \int \Delta C \mathrm{~d}(\ln T),
$$

where $\Delta H$ is the enthalpy change under constant temperature $T_{\mathrm{c}}$, which can happen during phase change, $\Delta S$ is the entropy change, and $\Delta C$ is the heat capacity change $\left(C_{\mathrm{s}}-C_{\mathrm{r}}\right)$. If $\Delta C$ is temperature independent during the temperature range, Eq. (3) can be simplified to Eq. (4).

$$
\Delta G=\Delta H\left(T_{\mathrm{c}}\right)-T \Delta S\left(T_{\mathrm{c}}\right)+\Delta C\left(T-T_{\mathrm{c}}-T \ln \frac{T}{T_{\mathrm{c}}}\right) .
$$

If $\Delta G$ is negative, the reaction or transition will proceed spontaneously to an extent governed by the magnitude of $\Delta G$. Otherwise, $\Delta G$ specifies the energy needed to drive

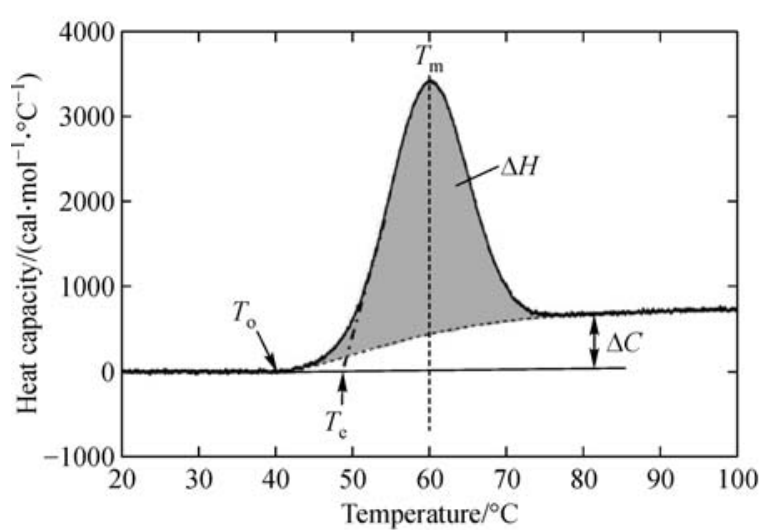

(a) the reaction to happen. The enthalpy change $\Delta H$ which is described in Eq. (5) reflects the energy needed to transfer the material to a new state. $T$ is the absolute temperature in Kelvin. The entropy can be simply described as an index to show how easily the energy might be distributed among various energy levels [28]. For protein binding reactions, the enthalpy is usually negative which reflects the tendency for the system to fall to lower energy levels by bond formation. In a DSC test, the enthalpy change $\Delta H$ can be directly obtained from the DSC curve shown in Fig. (3) [29], then the Gibbs free energy change is derived using Eq. (4).

$$
\Delta H=\Delta H\left(T_{\mathrm{c}}\right)+\Delta C\left(T-T_{\mathrm{c}}\right) .
$$

\section{Applications of DSC to biomolecular reactions}

In DSC, the temperature difference between sample and reference cells is monitored as the temperature of both is increased linearly in a precisely controlled manner. A thermally driven reaction in the sample cell leads to a temperature imbalance between the cells. When that occurs, power to the heaters on the cells is modulated to bring the sample and reference back into balance. The primary data recorded is compensation power as a function of temperature (power compensated DSC). Power, as a fundamental physical quantity, is readily converted to energy units of joules or calories per second. Figure 3 shows typical DSC curves of bimolecular reaction. Figure 3(a) shows the chicken hen lysozyme denaturation process. It is a typical one thermal domain process which is irreversible [30]. The grey area under the transition curve defines the enthalpy change during the reaction process. A difference between the pre- and post-transition baselines results if there is a change in heat capacity. The degree of

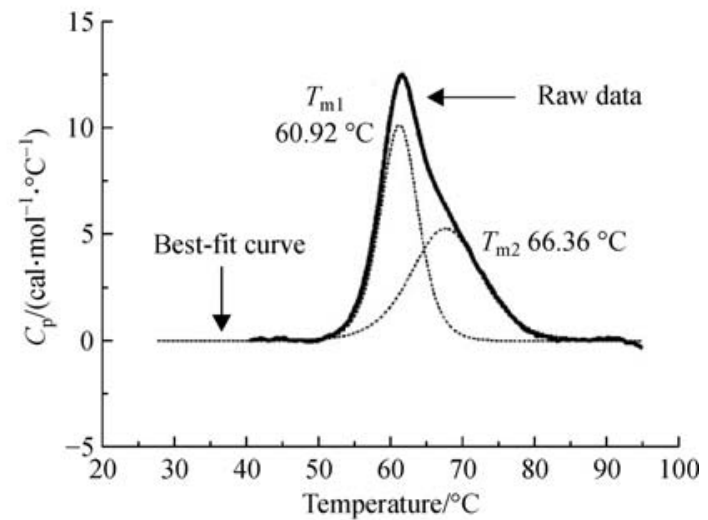

(b)

Fig. 3 Typical DSC curves of bimolecular reaction. (a) DSC thermogram for the hen lysozyme denaturation process; (b) schematic of the DSC thermogram observed for BSA denaturation process with the raw data and the separated two thermal domains after baseline subtraction $(1 \mathrm{kcal}=4.184 \mathrm{~kJ})[29]$ 
the transition at any temperature may be defined by integration of the transition curve. Such endothermic process can be described by the onset temperature, $T_{\mathrm{o}}$, the temperature where the transition starts to deviate from the baseline. The extrapolated onset temperature, $T_{\mathrm{e}}$, is the temperature at the intersection of extrapolated baseline prior to the transaction with extrapolated leading edge of the transaction [31]. $T_{\mathrm{m}}$ is often regarded as the melting point or the midpoint of the transaction. It is the peak temperature during the whole single thermal domain. In some case, the biomolecule has no significant heat capacity change before and after the reaction $(\Delta C \approx 0)$. In this case, the baseline for the endotherm is simply obtained by connecting the point at which the curve departs from the baseline of the thermogram to where it rejoins the baseline. However, in most cases, there is a significant heat capacity change before and after the reaction. In this case, we cannot simply connect the points from the beginning to the end of the reaction process in the thermogram. Baseline approximations such as sigmoidal baseline or extrapolation of the high or low temperature baseline $[32,33]$. Figure 3(b) shows the denaturation process of bovine serum albumin (BSA) using the MicroCal VPDSC. The thermogram of BSA contains 2 thermal domains which means two overlapping transactions happened in scanning temperature range from $40{ }^{\circ} \mathrm{C}$ to $90{ }^{\circ} \mathrm{C}$. The peak temperature for the first domain is $T_{\mathrm{m} 1}$ which is $60.92{ }^{\circ} \mathrm{C}$ while the peak temperature of the second domain $T_{\mathrm{m} 2}$ is $66.36{ }^{\circ} \mathrm{C}$. In such case, multiple thermal domains are overlapped during the temperature scanning process, the raw DSC data is in irregular shape. Each thermal domain means a unique transaction with independent thermal parameters $\left(\Delta H\right.$ and $\left.T_{\mathrm{m}}\right)$. There are different models developed to separate the mixed thermal domains by data fitting. The simplest model is the 2 -state with zero $\Delta C$. It only uses $T_{\mathrm{m}}$ and $\Delta H$ to do the deconvolution of the raw data. The domain separation of BSA denaturation utilizes this method since $\Delta C$ is zero.

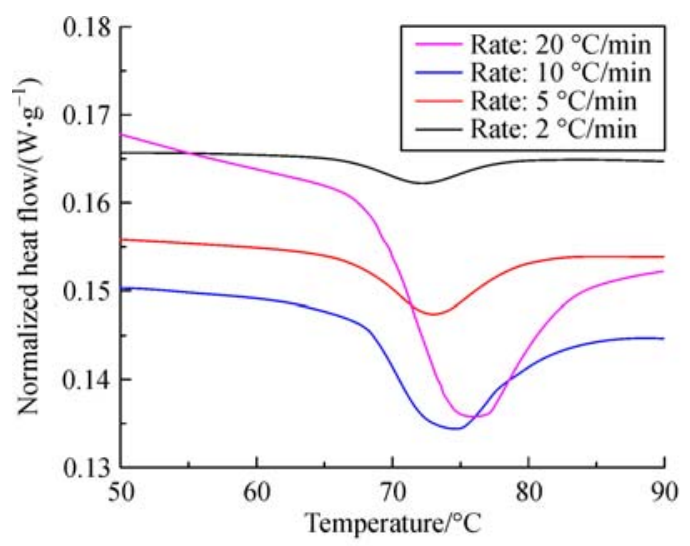

(a)
One defect of DSC is the studied sample would never be in complete thermal equilibrium due to the continuous heating strategy it applies. This limitation put certain requirements for the samples and the DSC itself as well. The temperature induced changes in the samples should not be too sharp and the relaxation time at these changes should be short. For the DSC, this requires the sample/ reference cell has minimal thermal gradient.

In irreversible denaturation process, an increase in scanning rate is expected to shift the melting point $T_{\mathrm{m}}$ [34]. A simple model that is consistent with irreversible protein denaturation is a reversible unfolding step followed by an irreversible process that locks the unfolded protein in a state from which it does not refold. Theoretically, the relationship between the scanning rate and the melting point can be illustrated by Eq. (6):

$$
\ln \left(\frac{\vartheta}{T_{\mathrm{m}}^{2}}\right)=\ln \left(\frac{A R}{E_{\mathrm{a}}}\right)-\frac{E_{\mathrm{a}}}{R T_{\mathrm{m}}},
$$

where $\vartheta$ is the scanning rate, $A$ is the empirical frequency factor which can be derived from the Arrhenius equation, $E_{\mathrm{a}}$ is the activation energy, $R$ is the gas constant. The slope of the plot $\ln \left(\frac{\vartheta}{T_{\mathrm{m}}^{2}}\right)$ as a function of $1 / T_{\mathrm{m}}$ equals $E_{\mathrm{a}} / R$.

In Fig. 4, the lysozyme sample is tested with the TA DSC 2500 under different scanning rate. The $y$ axis is the normalized differential heat flow. The scanning rate has a significant impact on the melting point of the lysozyme sample. The melting temperature almost shifts $6^{\circ} \mathrm{C}$ when the scanning rate is increased from 2 to $20^{\circ} \mathrm{C} / \mathrm{min}$.

\section{Development of MEMS based DSCs}

MEMS based calorimeter applies the standard microelectro-mechanical system technique to scale down the conventional benchtop size DSC to wafer or even smaller size. A typical MEMS DSC consists of three parts: The

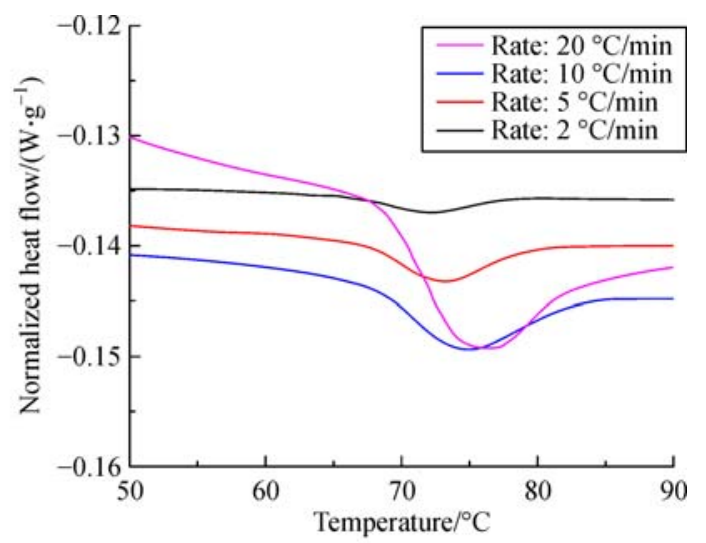

(b)

Fig. 4 The DSC curves for lysozyme sample with different scanning rate ranges from 2 to $20{ }^{\circ} \mathrm{C} / \mathrm{min}$. (a) DSC curves of lysozyme sample with the concentration $20 \mathrm{mg} / \mathrm{mL}$; (b) DSC curves of lysozyme sample with the concentration $10 \mathrm{mg} / \mathrm{mL}$ 
liquid deliver system, the thermal insulation structure, and the temperature sensing and heating unit. A MEMS DSC employs a liquid deliver system which is similar to the current commercial DSC (MicroCal VPDSC). The liquid deliver system is used to deliver the liquid sample and reference material to the thermal cells. It consists of high precision syringe pump, micro capillary and other connection parts. The thermal insulation structure includes the suspended thin film or polymer substrate with low thermal conductivity and microfluidic chamber integrated with air gap. The suspended substrate can be inorganic material such as $\mathrm{Si}_{3} \mathrm{~N}_{4}, \mathrm{SiO}_{2}$ or polymer such as SU8 or polyimide. There is a trend to use polymer to replace the traditional silicon complexes due to their thermal conductivity is usually one or two orders smaller. The microfluidic chamber is also fabricated using materials with low thermal conductivity such as polydimethylsiloxane (PDMS) or glass. Another requirement for such material is the high transparency which permits real time monitor of the temperature scanning process. The microfluidic chamber can be fabricated with the MEMS technique or using $3 \mathrm{D}$ printing with high resolution. The temperature sensing and heating unit is the core of the MEMS DSC. It consists of the temperature sensing and heating part. There are mainly two techniques to achieve temperature sensing with high sensitivity: Thermopile or thermistor. The function of the heating unit is to provide heat flux to the sample and reference material in the thermal cells. In such micro level heating with high thermal insulation, one big challenge is to minimize the thermal gradient in the heating material. Because these systems incorporate a very small thermal mass and use reagent quantities in the nanogram/nanoliter range, rapid and uniform heating and cooling can be achieved while maintaining a high level of temperature homogeneity. These miniaturized nano DSC can offer enhanced sensing capabilities in an inexpensive portable format.

Figure 5 [35-37] shows the challenge to fabricate the corresponding components in a MEMS DSC compared to the commercial DSC. The crucible to contain the sample and reference material is replaced by microfluidic chamber. The pedestal structure is abandoned, a suspended membrane provides substrate for the integrated heating and temperature sensing unit and the microfluidic chamber with excellent thermal isolation. Unlike the conventional DSC which utilize cumbersome heating/cooling block and the macro thermocouple for temperature scanning and sensing. In MEMS DSC, the micro heater and temperature sensor are fabricated on the suspended membrane directly using standard MEMS technique. The performance of a MEMS DSC heavily depends on these three parts: Microfluidic chamber, suspended membrane, and temperature sensing and heating unit. The improvement of MEMS DSC is achieved by integrating novel microfluidic chamber structure with better thermal insulation, smaller addenda thermal mass (heat capacity), robust suspended membrane with lower thermal conductivity, temperature sensing and heating unit with higher temperature sensitivity and more uniform temperature scanning.

\subsection{Thermal insulation system}

The simplest way to achieve high thermal insulation is using suspended membrane under high vacuum environ-

(a)

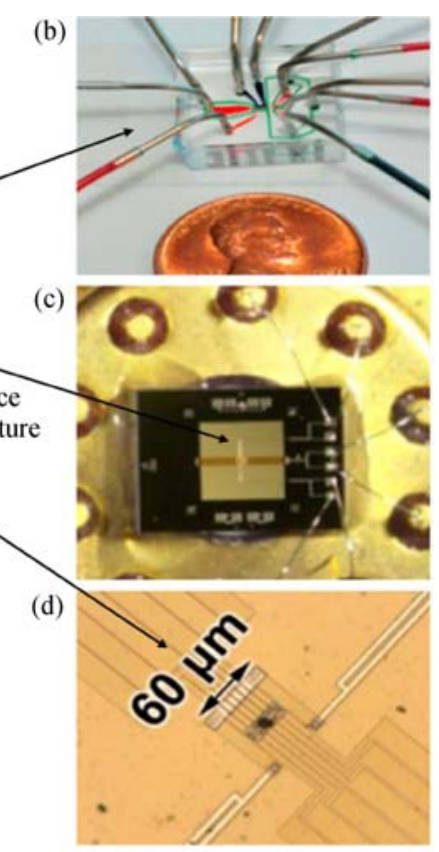

Fig. 5 Challenges to develop MEMS DSC compared to the conventional DSC. (a) Schematic set up for commercial macro DSC [35]; (b) microfluidic chamber device [36]; (c) suspended membrane for the MEMS DSC; (d) temperature sensing and heating unit [37] 
ment. Since biomolecules are often resolved in liquid, there is serious evaporation problem without careful chamber system during the temperature scanning process [38]. Hence the simple open type structure which is utilized by most MEMS isothermal titration calorimeters cannot be applied to the MEMS DSC [39,40]. A carefully designed microfluidic chamber system is designed to prevent the evaporation and provide high thermal insulation performance. The thermal insulation system for MEMS DSC consists two parts: The suspended membrane as the substrate for the temperature sensing and heating unit and the microfluidic device $[41,42]$.

Two types of the suspended membrane material are the silicon compound such as $\mathrm{SiO}_{2}$, polysilicon, $\mathrm{Si}_{3} \mathrm{~N}_{4}$ [43-45] and polymers [46-48]. Among the silicon compounds, $\mathrm{Si}_{3} \mathrm{~N}_{4}$ is the best for its total inertness and can be easily manipulated. Fabrication of membrane using $\mathrm{Si}_{3} \mathrm{~N}_{4}$ is mastered in all cleanroom in the world using chemical etching of silicon coated with silicon nitride on both side by $\mathrm{KOH}$ based etchant. Thermometer and heater can be lithographied on top of the silicon nitride membrane which permits the measurement of thermal properties. Polymer membranes are also used to insure good thermal insulation for MEMS DSC. In the temperature range applied to MEMS DSC (room temperature to $110^{\circ} \mathrm{C}$ for biomolecular study), polymers such as SU8, polyimide has unique advantages such as robustness, low cost, low thermal conductivity and inertness.

Microfluidic chamber is the essential part of the thermal insulation system for MEMS DSC. One method to fabricate the microfluidic chamber is to use Pyrex glass for its high transparency, low thermal conductivity, low cost and standard micro machining process [49]. There are several steps to fabricate the microfluidic chamber with Pyrex glass wafer.

1) Silicon etching. Using silicon wafer as the master mold, the desired cavities are obtained by wet etching or deep reactive ion etching etching.

2) Anodic bonding. After cleaning, the silicon wafer is hermetically anodic bonding to the Pyrex glass under high vacuum environment. Typically, $1000 \mathrm{~V}$ voltage is added between the silicon wafer and the glass wafer.

3) Heat treatment. In this step, the bonded wafer is heated up to the strain point of the Pyrex glass $\left(850^{\circ} \mathrm{C}\right)$ in a furnace under the standard atmospheric pressure. At this temperature, the Pyrex glass will be softened and fill the cavities on the silicon wafer.

4) Annealing. In this step, the bonded wafer is put into the heat treatment furnace to take an annealing process under the temperature of the annealing point of Pyrex glass.

5) Microfluidic device separation. After the annealing process, the Pyrex glass already forms the microfluidic chamber on the silicon wafer by filling the cavities. In this step, the residual silicon is removed by wet etching. Another popular method is the polydimethylsiloxane based microlithography technique.

There are several steps in such microfluidic chamber fabrication process [50,51].

1) SU8 patterning. SU8 is used to fabricate the master mold for the PDMS based microfluidic chamber. It is firstly spin coated on a cleaned silicon wafer and then patterned with standard photolithography technique to form the predesigned cavities.

2) PDMS casting. The surface of the SU8 is firstly treated under plasma for later release of the PDMS chamber. The PDMS is then mixed with the base and agent under proper ratio (10:1 in weight) [52]. After the PDMS casted to the silicon wafer, the whole device is put in a vacuum chamber for degassing.

3) Heat treatment. In this step, the device is put onto a hot plate to slowly increase the temperature to the curing temperature and hold for two hours (The curing temperature and time may vary depends on the expected properties of the PDMS chamber such as Young's modulus.).

4) PDMS microfluidic device release. After the curing, the PDMS chamber can be released from the silicon wafer by simply pilling off. The master pattern can then be cleaned and reused for the next fabrication. The fabrication process of these two methods are shown in Fig. 6.

After the fabrication of microfluidic chamber. One critical task is to bond the chamber to the suspended membrane of the DSC device. PDMS usually has very good adhesion with silicon, silicon nitride and silicon dioxide, however it has poor adhesion to polymers. In such case, additional adhesion layer is needed to increase the adhesion strength between the microfluidic chamber and the polymer membrane [53]. Figure 7 [26,48] shows two examples of the microfluidic system for MEMS calorimeters. In Fig. 7(a), the microfluidic system is fabricated with Pyrex glass. It consists of fluidic channel, sample inlet and outlet, the reaction chamber. This microfluidic system has thick glass walls contacted with the biomolecular sample which will contributes part of the heat capacity of the system and difficult to separate from the heat capacity of the sample. In Fig. 7(b), it utilizes Parylene to form thin wall $(2 \mu \mathrm{m})$ for the microfluidic chamber which can significantly reduce the additional heat capacity added to the sample. It has on-chip vacuum space which increases the thermal insulation of the calorimeter system in a further step.

\subsection{Temperature sensing and heating unit}

The sensitivity of a calorimeter is determined by the minimum thermal energy or power it can detect. In most cases, calorimetric measurement is achieved by measuring temperature changes [54]. Therefore, a high-sensitivity temperature sensor is needed in developing a highsensitivity calorimeter. There are mainly two temperature sensing methods in calorimetry: Thermopile and thermistor. Thermopiles (thermocouples connected in series) are 
(a)

(1) Silicon cleaning

(2) Silicon etching

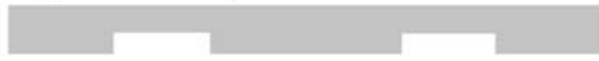

(3) Anodic bonding of silicon and Pyres glass

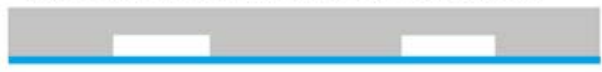

(4) Heat treatment and annealing

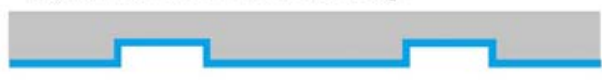

(5) Pyres glass chamber release

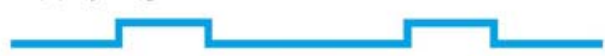

(b)

(1) Silicon cleaning

(2) SU8 spin coating

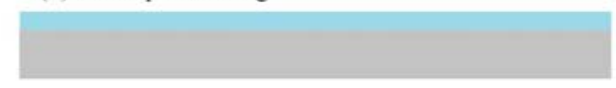

(3) SU8 patterning as the master mold

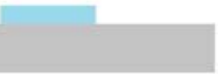

(4) PDMS filling

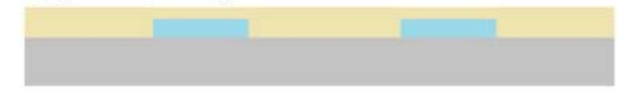

(5) PDMS chamber release

Fig. 6 Two typical microfluidic chamber fabrication method. (a) Glass based microfluidic chamber fabrication process; (b) PDMS microfluidic chamber fabrication process

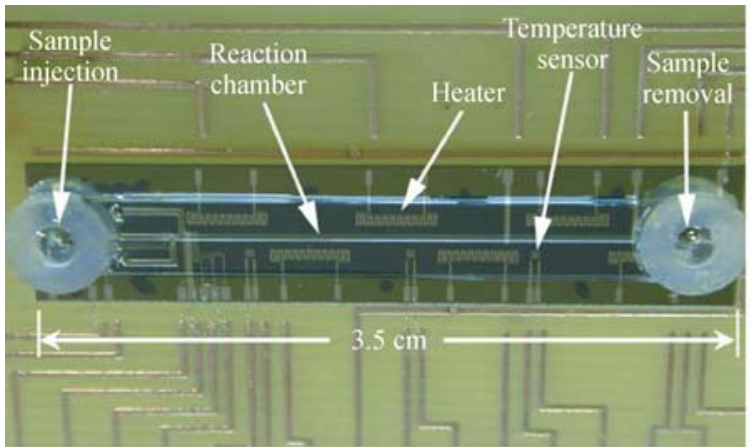

(a)

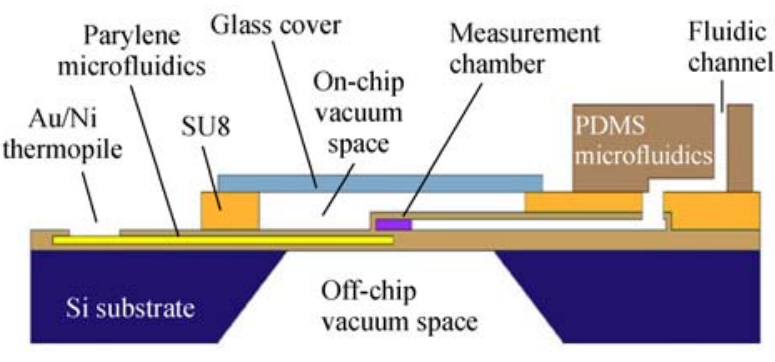

(b)

Fig. 7 (a) Microfluidic system for a novel nanocalorimeter [26]; (b) schematic view of the microfluidic system for a high sensitive microfluidic calorimeter [48]

used as thermometers in most MEMS DSCs [55-57]. The thermocouple converts the temperature difference to electric voltage via thermoelectricity. As thermopiles can have very high sensitivity by simply increasing the number of thermocouples and does not consume electric power to measure temperature, they are ideal for calorimetric applications. However, there are also limits for such method. First, the space will limit the number of thermocouples integrated in the thermopile. Second, the thermocouple usually has large resistance and they are connected in series, the electrical resistance will lead to large thermal noise. The other method is thermistor. The working principle of thermistor is the electrical resistance is related to temperature. Some semiconductor materials such as $\mathrm{SiC}, \mathrm{VO}_{x}$ have extremely high temperature coefficient of resistivity ( $-2 \%$ to $-5 \%$ per Kelvin) which are tens or even hundreds of times larger than the common metal materials $[58,59]$. The problem with thermistor is they need current for the temperature sensing which will generate additional Joule heat to the sample cells. Hence the current to the thermistor must be minimized for the temperature sensing or carefully controlled. Figure 8 $[47,60]$ shows two temperature sensing examples in calorimetry application

Micro heater design is another critical task for MEMS DSC. Although MEMS only needs milliwatts level of power to achieve the temperature scanning from room temperature to $100{ }^{\circ} \mathrm{C}$, consider the heating area is only in the square millimeter level, the heating power density would be high. The high thermal insulation of the calorimeter system will cause the temperature attenuates rapidly from the heating area to the non-heating area [61]. A good heater design should consider the thermal gradient in the heating cell will affect the test result and try to achieve high temperature uniformity in the heating area $[62,63]$. 


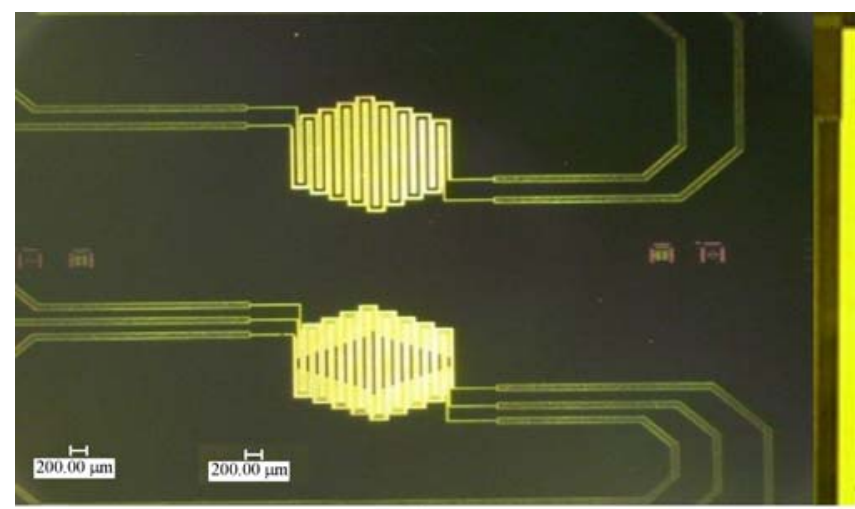

(a)

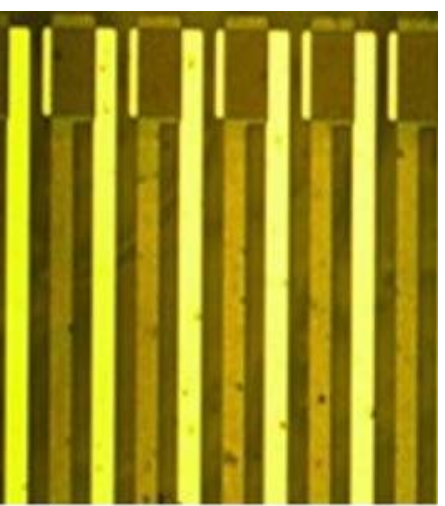

(b)

Fig. 8 Two temperature sensing examples in calorimetry application. (a) Four SiC thermistors form a Wheatstone bridge for the temperature measurement [47]; (b) 50 junctions of thermocouples are connected in series to build the thermopile to measure the differential temperature between the sample and reference cell [60]

\subsection{Application of MEMS DSC for biomaterial study}

MEMS DSC can be extended to apply for biomolecular study with less sample consumption, lower sample concentration and higher temperature scanning rate duo to its minimized size. One of the most important parameters of a MEMS DSC is its sensitivity measured by the corresponding output voltage to a certain heating power difference between the sample and reference cells $(\mathrm{V} / \mathrm{W})$. A typical sensitivity calibration method is to apply a certain amount of Joule heat to the sample cell (microwatts level) and leave the reference cell unheated or vice versa while both cells are filled with buffer. This requires the heating module can heat up the cell uniformly. By this method, we can obtain the continuous response to the different power difference by simply changing the current applied to the micro heater. Other methods such as the mixing of certain amount of acid and alkaline in the sample chamber while filling the reference cell with buffer to produce heat can also be used to calibrate the sensitivity. This method can lead to more

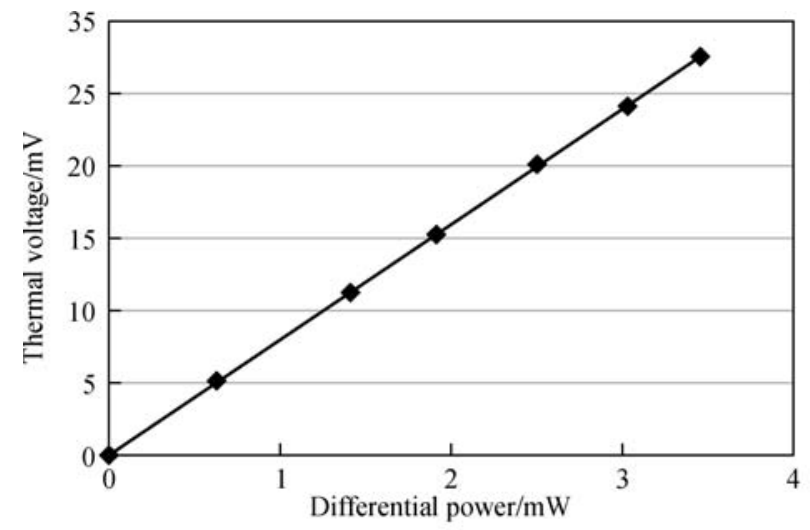

(a) accurate sensitivity calibration since it uses chemical reaction instead of Joule heat to produce the power difference which is closer to the real test. However, this method also requires precise reaction rate control to generate constant power for stable output which will make the system too complicated. Figure 9(a) [64] shows the sensitivity calibration of a MEMS DSC using Joule heat. The sensitivity of the MEMS DSC is $7 \mathrm{~V} / \mathrm{W}$ which is high enough to detect weak signal during the scanning process.

MEMS DSC can increase the scanning rate of liquid sample to $100{ }^{\circ} \mathrm{C} / \mathrm{s}$ or even higher by greatly reducing the total thermal mass of the DSC system which is impossible for traditional DSC. The super high scanning rate can be extremely useful to detect weak binding and lead to other discoveries. Figure 9(b) [65] shows one example of using a fast MEMS DSC for lactose study. Under high scanning rate $\left(100-500^{\circ} \mathrm{C} / \mathrm{min}\right)$ not only the melting temperature is shifted, but also the thermal domains which cannot be detected under lower scanning rate $\left(20^{\circ} \mathrm{C} / \mathrm{min}\right)$ is clearly shown in the diagram.

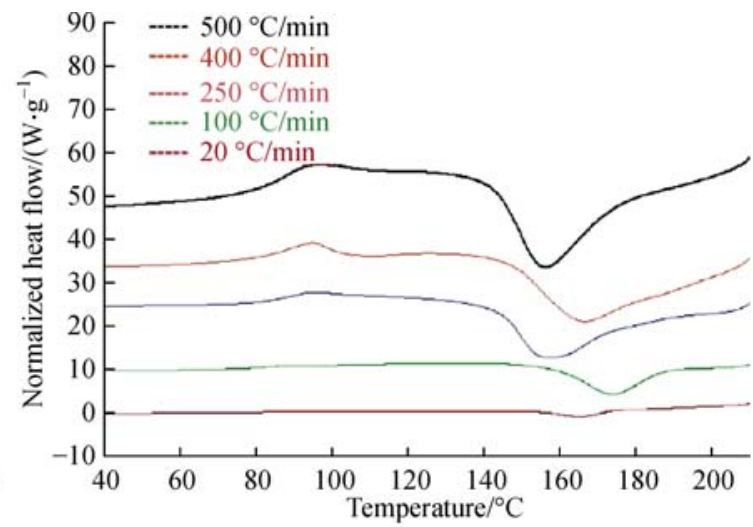

(b)

Fig. 9 (a) Sensitivity calibration of a MEMS DSC using Joule heat [64]; (b) DSC scans showing the analysis of spray-dried lactose at a variety of high heating rates using fast scan DSC [65] 


\subsection{Recent MEMS DSC research}

Currently, MEMS DSC are developed for high throughput calorimetry, super sensitive biochemical sensing and cellular level measurement applications. There are significant works done towards these goals which are listed in Table 1 [60,66-69]. They employ novel structures, temperature sensing materials to achieve high thermal performance. Some of these designs are show in Fig. 10 [64,66-68]. Figures 10(a) and 10(b) are MEMS DSC fabricated on suspended $\mathrm{Si}_{3} \mathrm{~N}_{4}$ membrane. The $\mathrm{Si}_{3} \mathrm{~N}_{4}$ membrane is only $100 \mathrm{~nm}$ in thickness with low yield strength. It is carefully designed to withstand much smaller sample volume nanoliter compared to the MEMS DSCs which utilize polymer based membrane which are shown in Figs. 10(c) and 10(d). They both use polyimide as the suspended membrane due to its robustness and simple fabrication process. The thickness of the polyimide membrane in around $10 \mu \mathrm{m}$ and it has much larger yield strength, hence, it can withstand larger sample volume (microliter level). For such small sample volume (nano/ microliter), the heat release during the denaturation process is extremely small. For such small signal detection, one requirement is super high temperature sensitivity and the other is ultraprecise thermal environment control since any undesired heat exchange of heat between the sample and

Table 1 Lists of most up to date MEMS DSC work

\begin{tabular}{|c|c|c|c|c|}
\hline MEMS DSC work & Sample consumption $/ \mathrm{nL}$ & Sensitivity $/\left(\mathrm{V} \cdot \mathrm{W}^{-1}\right)$ & Noise level in temperature $/ \mu \mathrm{K}$ & Noise level in power/nW \\
\hline Lin's group, 2015 [60] & 1000 & 4.78 & & 20 \\
\hline Lee's group, 2016 [66] & 30 & & 48.00 & 10 \\
\hline Lee's group, 2014 [67] & 200 & & 8.17 & \\
\hline Zuo's group, 2016 [68] & 1000 & 6.00 & 60.00 & 40 \\
\hline Saito and Nakabeppu, 2015 [69] & Flow through & & & 100 \\
\hline
\end{tabular}

(a)
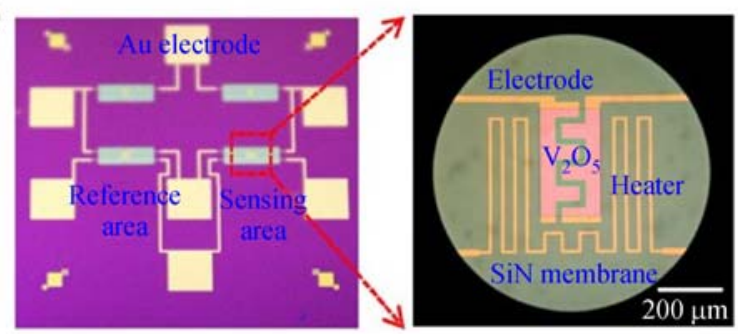

(c)

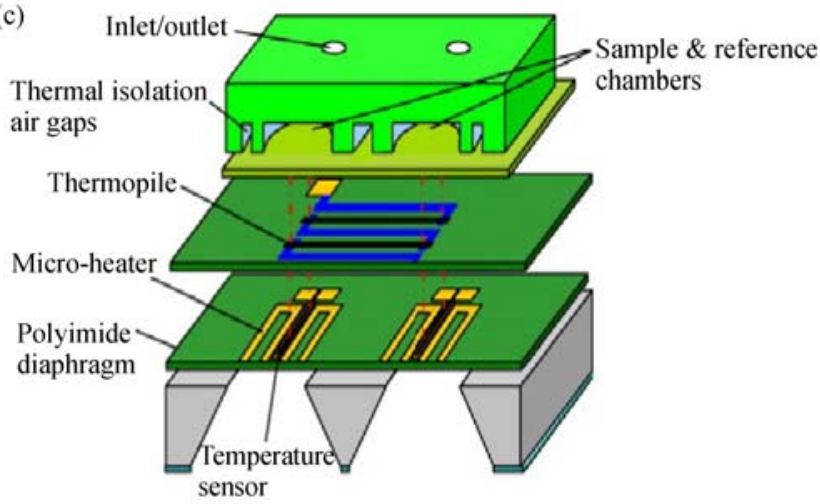

(b)

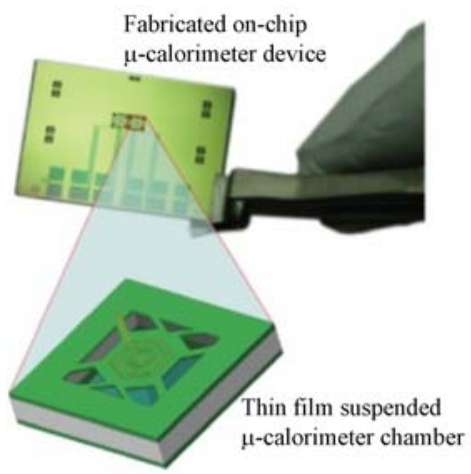

(d)

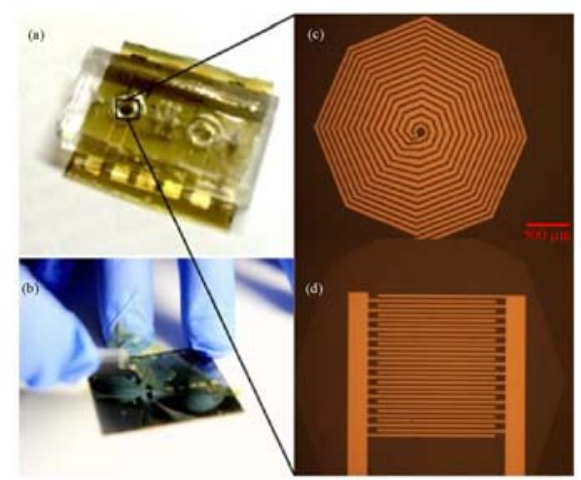

Fig. 10 Various examples of MEMS DSC designed for biomolecular study. (a) High sensitivity calorimeter platform using $\mathrm{V}_{2} \mathrm{O}_{5}$ thin film thermistor was developed. The calorimeter platform Integrates a $\mathrm{V}_{2} \mathrm{O}_{5}$ thermistor with a high temperature sensitivity of $-2.2 \% / \mathrm{K}$ with a vacuum insulated, suspended $\mathrm{SiN}$ membrane structure enabled a low thermal conductance of $12 \mu \mathrm{W} / \mathrm{K}$ and achieves direct detection of $10 \mathrm{nW}$ [66]; (b) $\mu$-calorimeter designed for the efficient coupling and detection of heat from the thermal elements for accurate characterization [67]; (c) MEMS DSC combines highly sensitive thermoelectric sensing, on-chip self-calibration, and microfluidic regulation for thermodynamic characterization of biomolecular samples on a minimized scale [64]; (d) MEMS DSC design integrated vanadium oxide thermistors and flexible polymer substrates with microfluidics chambers for ultrasensitive biomolecular study [68] 
the environment will generate random signal which can affect the result. Figures 10(a) and 10(b) employ vanadium oxide based thermistor as the temperature sensor. The vanadium based thermistor has high temperature coefficient of resistivity $(-2.5 \%)$. They use Wheatstone bridge to transfer resistance change caused by temperature difference to the output voltage signal. The Wheatstone bridge can effectively reject the common mode noise in the reference and sample cells such as temperature fluctuation and evaporation. In Fig. 10(c), antimony-bismuth (Sb-Bi) thermopile is embedded in the diaphragm for sensitive thermoelectric detection. Antimony (Seebeck coefficient: $43 \mu \mathrm{V} / \mathrm{K}$ ) and bismuth (Seebeck coefficient: $-79 \mu \mathrm{V} / \mathrm{K}$ ) are chosen for differential temperature sensing due to their high thermoelectric sensitivities and easy fabrication using standard MEMS technique. The 50-junction thermopile has a temperature sensitivity of $6.3 \mathrm{mV} /{ }^{\circ} \mathrm{C}$. In Fig. 10(b), the reaction chambers have been designed and fabricated using a $\mathrm{Si}_{x} \mathrm{~N}_{y}$ thin film and a thin polyimide film. The reaction chamber is also fully suspended from silicon handle to minimize the thermal loss to the substrate by thermal conduction. The reaction chamber has been 3Dmicromachined using an anisotropic wet chemical etching process. This method eliminates the off-chip wafer bonding processes and keeps the device fabrication robust and simple, and achieves low thermal mass and high thermal insulation result in high sensitivity of detection.

\section{Conclusions}

In biomolecular study and early drug design, cell based screening assays are used as biologically relevant surrogates. Compared to the cell based assays, DSC enables determination and understanding of the protein-ligand binding in a more direct and reliable way. MEMS DSC attracts growing interest due to the development of new capabilities, such as high-throughput array operations and microfluidics with high thermal insulation. This paper gives an overview of the DSC techniques in the application of biomolecular studies and the effort to develop MEMS based DSC. The unique capability of differential calorimetric sensing is highly expected to open an unconventional biosensor field, and the improved sensitivity and throughput of MEMS will permit applications in new research fields. Calorimetric techniques are widely used for material characterization. The outstanding work listed show the possibility of applying MEMS DSC to achieve smaller sample consumption, higher throughput in biomolecular study, drug design and bioprocess monitoring. A reasonable perspective of the MEMS DSC is to increase the noise level to picowatt to achieve single cell activity monitoring.

Acknowledgements The authors thank the funding support from NSF (IDBR \#1530508) and Abbvie Inc. The authors also thank Dr. Fernando Camino for the discussion.
Open Access This article is distributed under the terms of the Creative Commons Attribution 4.0 International License (http://creativecommons.org/ licenses/by/4.0/), which permits unrestricted use, distribution, and reproduction in any medium, provided you give appropriate credit to the original author(s) and the source, provide a link to the Creative Commons license, and indicate if changes were made.

\section{References}

1. Watson E S, O’Neill M J. US Patent 3263484, 1966-08-02

2. Turi Edith. Thermal Characterization of Polymeric Materials. Morristown: Elsevier, 2012

3. Wunderlich B, Jin Y, Boller A. Mathematical description of differential scanning calorimetry based on periodic temperature modulation. Thermochimica Acta, 1994, 238: 277-293

4. Coleman N J, Craig D Q M. Modulated temperature differential scanning calorimetry: A novel approach to pharmaceutical thermal analysis. International Journal of Pharmaceutics, 1996, 135(1-2): 13-29

5. Simon S L. Temperature-modulated differential scanning calorimetry: Theory and application. Thermochimica Acta, 2001, 374(1): $55-71$

6. Chaires J B. Calorimetry and thermodynamics in drug design. Annual Review of Biophysics, 2008, 37(1): 135-151

7. Leng F, Priebe W, Chaires J B. Ultratight DNA binding of a new bisintercalating anthracycline antibiotic. Biochemistry, 1998, 37(7): 1743-1753

8. Bruylants G, Wouters J, Michaux C. Differential scanning calorimetry in life science: Thermodynamics, stability, molecular recognition and application in drug design. Current Medicinal Chemistry, 2005, 12(17): 2011-2020

9. Gill P, Moghadam T T, Ranjbar B. Differential scanning calorimetry techniques: Applications in biology and nanoscience. Journal of Biomolecular Techniques, 2010, 21(4): 167-193

10. Lavoisier A L, Laplace P S, Guerlac H. Memoir on heat. Journal of the History of Biology, 1983, 16(3): 444-445

11. Collins K D. Charge density-dependent strength of hydration and biological structure. Biophysical Journal, 1997, 72(1): 65-76

12. Ahrer K, Buchacher A, Iberer G, et al. Thermodynamic stability and formation of aggregates of human immunoglobulin $\mathrm{G}$ characterised by differential scanning calorimetry and dynamic light scattering. Journal of Biochemical and Biophysical Methods, 2006, 66(1-3): 73-86

13. Chaires J B. Energetics of anthracycline-DNA interactions. In: Demeunynck M, Bailly C, Wilson W D, eds. Small Molecule DNA and RNA Binders: From Synthesis to Nucleic Acid Complexes. Hoboken: John Wiley \& Sons, Inc., 2004, 461-481

14. Malvern. Retrieved from https://www.copybook.com/media/pharmaceutical/profiles/canadian-orthopaedic-association/migrated/ images/Microcal-VP-Capillary-DSC-System-2a.jpg

15. Malvern. Retrieved from http://www.news-medical.net/image.axd? picture $=2014 \% 2 \mathrm{~F} 11 \% 2 \mathrm{FDiscovery}-\mathrm{DSC}$.jpg

16. Torres F E, Recht M I, Coyle J E, et al. Higher throughput calorimetry: Opportunities, approaches and challenges. Current Opinion in Structural Biology, 2010, 20(5): 598-605 
17. Chancellor E B, Wikswo J P, Baudenbacher F, et al. Heat conduction calorimeter for massively parallel high throughput measurements with picoliter sample volumes. Applied Physics Letters, 2004, 85(12): 2408-2410

18. Recht M I, De Bruyker D, Bell A G, et al. Enthalpy array analysis of enzymatic and binding reactions. Analytical Biochemistry, 2008, 377(1): 33-39

19. Higuera-Guisset J, Rodriguez-Viejo J, Chacon M, et al. Calorimetry of microbial growth using a thermopile based microreactor. Thermochimica Acta, 2005, 427(1-2): 187-191

20. Wang L, Zhao Y, Ng E, et al. A MEMS differential calorimeter for biomolecular characterization. In: Proceedings of IEEE International Conference on Micro Electro Mechanical Systems (MEMS). IEEE, 2005, 814-817

21. Cerdeiriña C A, Miguez J A, Carballo E, et al. Highly precise determination of the heat capacity of liquids by DSC: Calibration and measurement. Thermochimica Acta, 2000, 347(1-2): 37-44

22. McGregor C, Saunders M H, Buckton G, et al. The use of highspeed differential scanning calorimetry (Hyper-DSC ${ }^{\mathrm{TM}}$ ) to study the thermal properties of carbamazepine polymorphs. Thermochimica Acta, 2004, 417(2): 231-237

23. Price D M, Reading M, Hammiche A, et al. Micro-thermal analysis: Scanning thermal microscopy and localised thermal analysis. International Journal of Pharmaceutics, 1999, 192(1): 85-96

24. Torres F E, Kuhn P, De Bruyker D, et al. Enthalpy arrays. Proceedings of the National Academy of Sciences of the United States of America, 2004, 101(26): 9517-9522

25. Recht M I, Torres F E, De Bruyker D, et al. Measurement of enzyme kinetics and inhibitor constants using enthalpy arrays. Analytical Biochemistry, 2009, 388(2): 204-212

26. Liu Y S, Ugaz V M, Rogers W J, et al. Development of an advanced nanocalorimetry system for material characterization. Journal of Loss Prevention in the Process Industries, 2005, 18(3): 139-144

27. Connelly P R. Acquisition and use of calorimetric data for prediction of the thermodynamics of ligand-binding and folding reactions of proteins. Current Opinion in Biotechnology, 1994, 5(4): 381-388

28. Ren J, Jenkins T C, Chaires J B. Energetics of DNA intercalation reactions. Biochemistry, 2000, 39(29): 8439-8447

29. Kang F, Singh J. Conformational stability of a model protein (bovine serum albumin) during primary emulsification process of PLGA microspheres synthesis. International Journal of Pharmaceutics, 2003, 260(1): 149-156

30. Matthyssens G E, Simons G, Kanarek L. Study of the thermaldenaturation mechanism of hen egg-white lysozyme through proteolytic degradation. European Journal of Biochemistry, 1972, 26(4): 449-454

31. Clas S D, Dalton C R, Hancock B C. Differential scanning calorimetry: Applications in drug development. Pharmaceutical Science \& Technology Today, 1999, 2(8): 311-320

32. Richardson M J. Quantitative aspects of differential scanning calorimetry. Thermochimica Acta, 1997, 300(1-2): 15-28

33. Sarge S M, Hemminger W, Gmelin E, et al. Metrologically based procedures for the temperature, heat and heat flow rate calibration of DSC. Journal of Thermal Analysis, 1997, 49(2): 1125-1134

34. Vermeer A W P, Norde W. The thermal stability of immunoglo- bulin: Unfolding and aggregation of a multi-domain protein. Biophysical Journal, 2000, 78(1): 394-404

35. Steinmann W, Walter S, Beckers M, et al. Thermal analysis of phase transitions and crystallization in polymeric fibers. In: Elkordy A A, ed. Applications of Calorimetry in a Wide Context-Differential Scanning Calorimetry, Isothermal Titration Calorimetry and Microcalorimetry. Rijeka: INTECH Open Access Publisher, 2013

36. San-Miguel A, Lu H. Microfluidics as a tool for C. elegans research. WormBook: The Online Review of C. Elegans Biology, 2005, 1-19

37. Zhuravlev E, Schick C. Fast scanning power compensated differential scanning nano-calorimeter: 1 . The device. Thermochimica Acta, 2010, 505(1-2): 1-13

38. Johannessen E A, Weaver J M R, Bourova L, et al. Micromachined nanocalorimetric sensor for ultra-low-volume cell-based assays. Analytical Chemistry, 2002, 74(9): 2190-2197

39. Keller S, Vargas C, Zhao H, et al. High-precision isothermal titration calorimetry with automated peak-shape analysis. Analytical Chemistry, 2012, 84(11): 5066-5073

40. Gourishankar A, Shukla S, Ganesh K N, et al. Isothermal titration calorimetry studies on the binding of DNA bases and PNA base monomers to gold nanoparticles. Journal of the American Chemical Society, 2004, 126(41): 13186-13187

41. Johannessen E A, Weaver J M R, Cobbold P H, et al. A suspended membrane nanocalorimeter for ultralow volume bioanalysis. IEEE Transactions on Nanobioscience, 2002, 1(1): 29-36

42. Olson E A, Yu Efremov M, Kwan A T, et al. Scanning calorimeter for nanoliter-scale liquid samples. Applied Physics Letters, 2000, 77 (17): 2671-2673

43. Sarro P M, van Herwaarden A W, van der Vlist W. A silicon-silicon nitride membrane fabrication process for smart thermal sensors. Sensors and Actuators A: Physical, 1994, 42(1-3): 666-671

44. Zhang Y, Tadigadapa S. Calorimetric biosensors with integrated microfluidic channels. Biosensors and Bioelectronics, 2004, 19(12): 1733-1743

45. Dijkstra M, de Boer M J, Berenschot J W, et al. Miniaturized flow sensor with planar integrated sensor structures on semicircular surface channels. In: Proceedings of IEEE 20th International Conference on Micro Electro Mechanical Systems (MEMS). IEEE, 2007, 123-126

46. Yin J, Yu S, Wang S, et al. Design and fabrication of flexible differential scanning nanocalorimeter. In: Proceedings of ASME 2014 International Design Engineering Technical Conferences and Computers and Information in Engineering Conference. New York: American Society of Mechanical Engineers, 2014, V004T09A002

47. Lei L, Chen X, Yu S, et al. Design and fabrication of a differential scanning nanocalorimeter. Journal of Micromechanics and Microengineering, 2016, 27(2): 025006

48. Lee W, Fon W, Axelrod B W, et al. High-sensitivity microfluidic calorimeters for biological and chemical applications. Proceedings of the National Academy of Sciences of the United States of America, 2009, 106(36): 15225-15230

49. Liu J, Huang Q, Shang J, et al. Micromachining of Pyrex 7740 glass and their applications to wafer-level hermetic packaging of MEMS devices. In: IEEE 23rd International Conference on Micro Electro Mechanical Systems (MEMS). IEEE, 2010, 496-499

50. Blake A J, Pearce T M, Rao N S, et al. Multilayer PDMS 
microfluidic chamber for controlling brain slice microenvironment. Lab on a Chip, 2007, 7(7): 842-849

51. Wang L, Lei L, Ni X F, et al. Patterning bio-molecules for cell attachment at single cell levels in PDMS microfluidic chips. Microelectronic Engineering, 2009, 86(4-6): 1462-1464

52. Johnston I D, McCluskey D K, Tan C K L, et al. Mechanical characterization of bulk Sylgard 184 for microfluidics and microengineering. Journal of Micromechanics and Microengineering, 2014, 24(3): 035017

53. Li X, Wu N, Rojanasakul Y, et al. Selective stamp bonding of PDMS microfluidic devices to polymer substrates for biological applications. Sensors and Actuators A: Physical, 2013, 193: 186192

54. Lee W, Lee J, Koh J. Development and applications of chip calorimeters as novel biosensors. Nanobiosensors in Disease Diagnosis, 2012, 1: 17-29

55. Baier V, Fdisch R, Ihring A, et al. Highly sensitive thermopile heat power sensor for micro-fluid calorimetry of biochemical processes. Sensors and Actuators A: Physical, 2005, 123-124: 354-359

56. Lerchner J, Wolf A, Wolf $\mathrm{G}$, et al. A new micro-fluid chip calorimeter for biochemical applications. Thermochimica Acta, 2006, 445(2): 144-150

57. Huynh T P, Zhang Y, Yehuda C. Fabrication and characterization of a multichannel 3D thermopile for chip calorimeter applications. Sensors (Basel), 2015, 15(2): 3351-3361

58. Han Y H, Kim K T, Shin H J, et al. Enhanced characteristics of an uncooled microbolometer using vanadium-tungsten oxide as a thermometric material. Applied Physics Letters, 2005, 86(25): 254101

59. Wang B, Lai J, Li H, et al. Nanostructured vanadium oxide thin film with high TCR at room temperature for microbolometer. Infrared Physics \& Technology, 2013, 57: 8-13

60. Jia Y, Wang B, Zhang Z, et al. A polymer-based MEMS differential scanning calorimeter. Sensors and Actuators A: Physical, 2015, 231: 1-7

61. Yu S, Wang S, Lu M, et al. A novel micro heater integrated on flexible polyimide substrate with fast response and uniform temperature distribution. In: Proceedings of ASME 2015 International Design Engineering Technical Conferences and Computers and Information in Engineering Conference. New York: American Society of Mechanical Engineers, 2015, V004T09A023

62. Lee S M, Dyer D C, Gardner J W. Design and optimisation of a high-temperature silicon micro-hotplate for nanoporous palladium pellistors. Microelectronics Journal, 2003, 34(2): 115-126

63. Carreto-Vazquez V H, Liu Y S, Bukur D B, et al. Chip-scale calorimeters: Potential uses in chemical engineering. Journal of Loss Prevention in the Process Industries, 2011, 24(1): 34-42

64. Wang B, Lin Q. Temperature-modulated differential scanning calorimetry in a MEMS device. Sensors and Actuators B: Chemical, 2013, 180: 60-65

65. Ford J L, Mann T E. Fast-scan DSC and its role in pharmaceutical physical form characterisation and selection. Advanced Drug Delivery Reviews, 2012, 64(5): 422-430

66. Koh J, Lee W, Shin J H. High-sensitivity chip calorimeter platform for sub-nano watt thermal measurement. Sensors and Actuators A: Physical, 2016, 241: 60-65

67. Davaji B, Jeong Bak H, Chang W J, et al. A novel on-chip threedimensional micromachined calorimeter with fully enclosed and suspended thin-film chamber for thermal characterization of liquid samples. Biomicrofluidics, 2014, 8(3): 034101

68. Wang S, Yu S, Siedler M S, et al. Micro-differential scanning calorimeter for liquid biological samples. Review of Scientific Instruments, 2016, 87(10): 105005

69. Saito M, Nakabeppu O. Flow type bio-chemical calorimeter with micro differential thermopile sensor. Journal of Nanoscience and Nanotechnology, 2015, 15(4): 2917-2922 